\title{
H-type hypertension and risk of stroke in chinese adults: A prospective, nested case- control study
}

\author{
Jianping Li ${ }^{1, \S}$, Shanqun Jiang ${ }^{2,3, \S}$, Yan Zhang ${ }^{1}$, Genfu Tang ${ }^{3}$, Yu Wang ${ }^{3}$, \\ Guangyun $\mathrm{Mao}^{3}$, Zhiping $\mathrm{Li}^{3}$, Xiping $\mathrm{Xu}^{3,4}$, *Binyan Wang ${ }^{4}$, *Yong Huo ${ }^{1}$ \\ 'Department of Cardiology, Peking University First Hospital, Beijing, China; \\ ${ }^{2}$ School of Life Sciences, Anhui University, Hefei, China; \\ ${ }^{3}$ Institute of Biomedicine, Anhui Medical University, Hefei, China; \\ ${ }^{4}$ National Clinical Research Study Center for Kidney Disease; State Key Laboratory for Organ Failure Research; \\ Renal Division, Nanfang Hospital, Southern Medical University, Guangzhou, China
}

\$These two authors contributed equally to this paper.

${ }^{*}$ Address for Correspondence:

Binyan Wang, M.D., Ph.D.

National Clinical Research Center for

Kidney Disease; State Key Laboratory for

Organ Failure Research; Renal Division,

Nanfang Hospital, Southern Medical

University, Guangzhou, China

Email: binyanwang126@126.com

Yong Huo, MD

Department of Cardiology, Peking

University First Hospital,

Beijing, China

Email: huoyong@263.net.cn

\begin{tabular}{|l|}
\hline Access this article online \\
\hline Website: \\
www.intern-med.com \\
\hline DOI: \\
10.1515/jtim-2015-0027 \\
\hline Quick Response Code: \\
\hline \\
\end{tabular}

\section{ABSTRACT}

Objectives: To investigate the independent and joint associations of hyperhomocysteinemia and hypertension with incident stroke and stroke death in Chinese adults. Methods: About 39,165 rural Chinese adults aged 35 years or older who had no history of stroke at the baseline study were prospectively followed to determine major cardiovascular events, with an average follow-up of 6.2 years. Using a nested case-control design, this report includes 179 incident stroke cases (121 stroke deaths) and 179 controls without vascular events from the original cohort matched by age, sex, community, and length of plasma storage. Baseline plasma total homocysteine (tHcy) measurements were obtained for all subjects. Logistic regression analysis was performed to investigate the independent and joint associations between $\mathrm{H}$-type hypertension, defined as subjects with concomitant hypertension and elevated homocysteine $(\geq 10 \mu \mathrm{mol} / \mathrm{L})$, and risk of incident stroke and stroke death, after adjusting for important covariates. Results: We analyzed each risk factor independently and jointly. For analysis, homocysteine was divided into three groups: low (tHcy $<10 \mu \mathrm{mol} / \mathrm{L})$, moderate $(\geq 10$ $\mu \mathrm{mol} / \mathrm{L} \mathrm{tHcy}<20 \mu \mathrm{mol} / \mathrm{L}$ ), and high ( $\mathrm{tH} c y \geq 20 \mu \mathrm{mol} / \mathrm{L}$ ). Compared to subjects in the low group, the odds ratios $(95 \% \mathrm{Cl})$ of incident stroke for those in the moderate group and the high group were $1.7(0.8-3.7)$ and $3.1(1.2-8.6)$, respectively. The odds ratios $(95 \% \mathrm{Cl})$ of stroke death for the moderate and high groups were $2.8(1.1-7.4)$ and 5.1 (1.6-16.4), respectively. Hypertension was also independently associated with a higher risk of incident stroke and stroke death: $3.8(2.3-6.4)$ and $3.2(1.8-6.0)$, respectively, compared to those without hypertension. When analyzed jointly, the highest risk was found among patients with $\mathrm{H}$-type hypertensive with both hyperhomocysteinemia and hypertension: 12.7 (2.8-58.0) for incident stroke and 11.7 (2.5-54.7) for stroke death. Conclusions: This study provides strong evidence that hyperhomocysteinemia and hypertension are two independent, modifiable risk factors, which act additively to increase the risk of incident stroke and stroke death. The results strongly suggest that $\mathrm{H}$-type hypertension is a major risk factor for vascular disease and mortality, and those with $\mathrm{H}$-type hypertension may particularly benefit from homocysteine-lowering therapy along with anti-hypertension therapy in Chinese populations.

Key words: H-type hypertension, stroke, nested case-control study, synergistic effect

\section{INTRODUCTION}

A number of epidemiologic and clinical studies have suggested that homocysteine (Hcy) levels are associated with vascular diseases including coronary artery disease (CAD), peripheral artery disease, and stroke ${ }^{[1-11]}$. However, results have been conflicting because some reports have shown a negative association between elevated plasma Hcy levels and stroke subtypes ${ }^{[12-15]}$. It is well known that the conclusions from several 
well-established meta-analyses ${ }^{[16-18]}$ that found an association between moderate elevation of Hcy levels and increased risk of vascular disease were mostly based on findings from studies of a cross-sectional or retrospective casecontrol design. Thus, it is difficult to determine the extent to which hyperhomocysteinemia (elevated Hcy levels) may be a precursor to or a consequence of vascular disease. Furthermore, most previous studies were conducted in western populations; it remains to be determined whether the associations found in western populations can be applied to Chinese populations. To date, only three prospective studies on Asian populations ${ }^{[12,13,19]}$ have assessed the association between plasma Hcy levels and vascular disease risk, but their conclusions are controversial. More importantly, no prospective study has assessed the interaction effect of hyperhomocysteinemia on other established risk factors that may affect pathogenesis and development of vascular events. For example, hypertension is a well-established risk factor for vascular disease ${ }^{[20-24]}$; however, previous studies have not examined the potential interaction between hyperhomocysteinemia and hypertension in the occurrence and development of vascular disease.

This study, using a nested case-control design, was designed to investigate the independent and joint associations of hyperhomocysteinemia and hypertension with incident stroke and stroke death in a large cohort of Chinese adults aged 30 years or older $(n=39,165)$, prospectively followed for an average length of 6.2 years.

\section{METHODS}

\section{Subjects}

A total of 39,165 subjects participated in an epidemiologic study of chronic diseases, conducted from 1995 to 2005 in nine communities of Anqing, Anhui Province, China. The study protocol was approved by the Ethics Committee of the Biomedical Institute of Anhui Medical University, China. Written informed consent was obtained from each study subject. Residents from one of the nine communities who were aged 35 years or older were eligible to participate in the study. Subjects were excluded if they had a history of severe chronic cardiac failure, coronary heart disease, myocardial infarction, acute coronary syndrome, severe cardiac arrhythmia, unstable angina, stroke, renal insufficiency, impaired liver function, or tumor. Subjects who were pregnant or lactating were also excluded.

\section{Baseline data collection}

Local village doctors assisted with subject recruitment. Subjects were invited to the study center and stayed there overnight before the baseline study. All subjects fasted overnight, and the following morning each completed the following procedures. Between 7 and 8 AM, a sample of antecubital venous blood was drawn into an
EDTA (ethylenediaminetetraacetic acid) blood collection tube. Blood pressure was recorded in the morning (after at least $8 \mathrm{~h}$ of supine rest) by two auscultation apparatuses simultaneously. A standardized questionnaire interview was then conducted to obtain information on age, gender, smoking habit, alcohol drinking, and relevant family medical history such as angina pectoris, hypertension, diabetes mellitus, myocardial infarction, premature coronary heart disease (documented coronary heart disease in at least one first-degree relative before the age of 55 years for men or 60 years for women), noncardiovascular diseases, drug treatment, and adherence to a lipid-lowering diet. Anthropometric parameters such as height and weight were measured using standardized methods on the same day.

\section{Follow-up and definition of vascular end points}

Subjects who participated in the baseline study were resurveyed with a mean follow-up interval of 6.2 years. With assistance from local village doctors, subjects received a follow-up visit where a second standardized questionnaire interview was conducted on each subject by trained research staff to obtain information on vascular end points, including angina pectoris, hypertension, diabetes mellitus, myocardial infarction, cardiogenic sudden death, renal insufficiency, and stroke, as well as type of hospital that made the diagnosis. Follow-up data was completed for 39,036 subjects, and 129 subjects were lost to follow-up because of relocation.

This report focuses on incident stroke and stroke death. Incident stroke was defined as a new stroke case occurring during the follow-up period, based on well-established physician diagnosis. Stroke death was defined as a death occurring during the follow-up period with stroke as the underlying cause of death, based on well-established physician diagnosis. Unfortunately, the distinction between cerebral infarction and cerebral hemorrhage could not be made because of a lack of imaging data for all stroke cases.

Over the course of follow-up, there were a total of 251 incident strokes (including 175 stroke deaths) identified from this cohort. This report includes 179 incident stroke cases (121 stroke deaths) who had both complete data and a baseline blood sample for the Hcy assay. The remaining 72 cases of incident stroke were excluded because of the lack of baseline blood samples. We selected 179 controls without any vascular events from the same cohort. The controls were matched to the stroke cases by age, gender, resident community, and length of plasma storage time. A sampling diagram of the study population is shown in Figure 1. We examined the baseline characteristics between the stroke cases $(n=179)$ included in the analyses and the stroke cases excluded from the analysis $(n=72)$ because of 


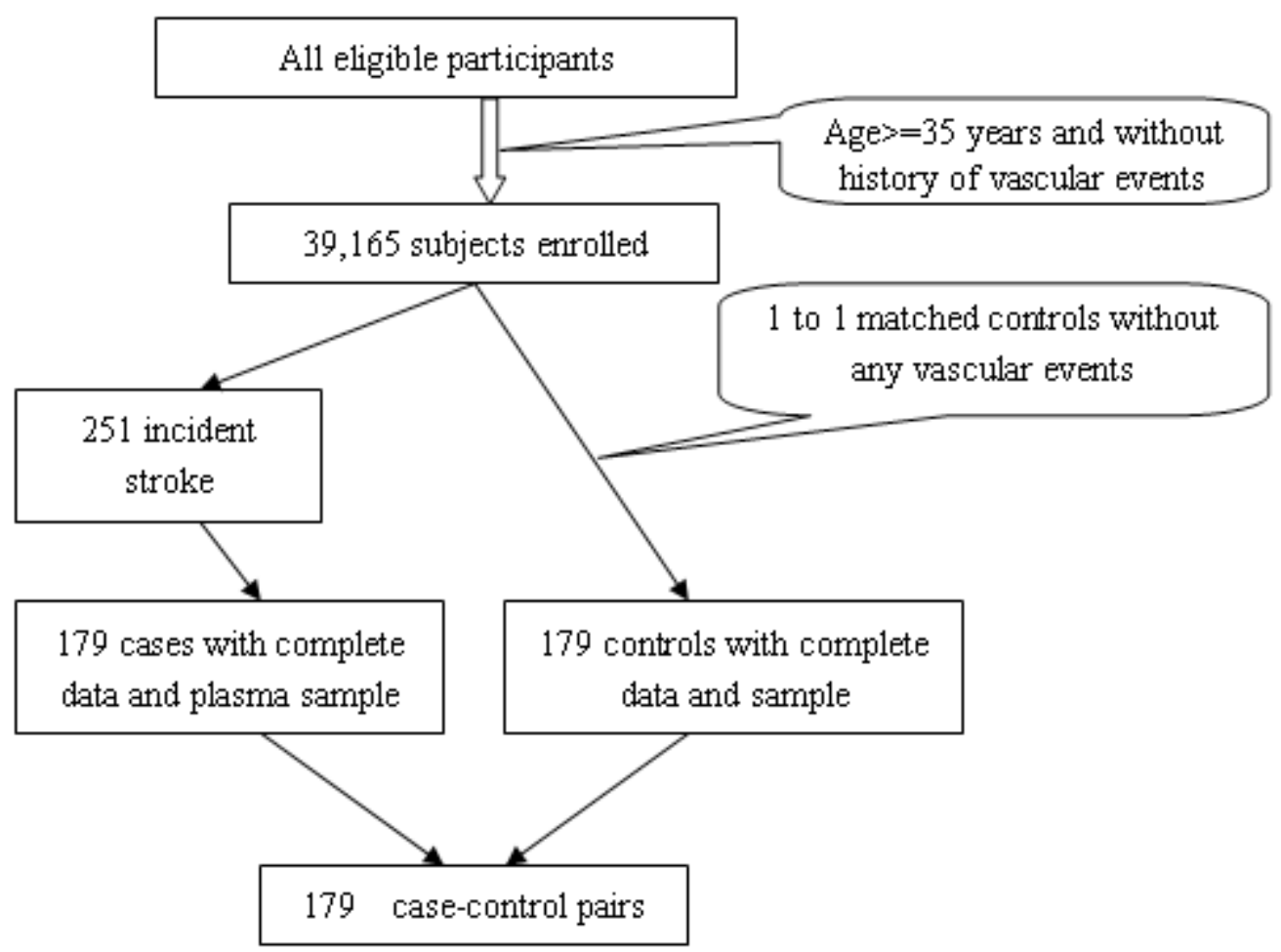

Figure 1. Diagram of studypopulation

the lack of baseline blood sample and found the two groups to be comparable at baseline in terms of age, gender, and body mass index (BMI) distribution (data not shown).

\section{Laboratory assays}

Plasma was separated from blood cells by centrifuge at $4^{\circ} \mathrm{C}$ (3000g for $10 \mathrm{~min}$ ) within $15 \mathrm{~min}$ of sample collection and stored in a $-80^{\circ} \mathrm{C}$ freezer until analysis. Fasting blood glucose, serum total cholesterol and triglycerides, highdensity lipoprotein, and low-density lipoprotein were measured within $2 \mathrm{~h}$ of sample collection using standard reagents and the same automatic biochemistry analyzer. All laboratory analyses were carried out by technicians who were blinded to the subjects' clinical status.

Plasma total homocysteine (tHcy), including free, bound, reduced, and oxidized forms of Hcy in the blood, was measured by high-performance liquid chromatography with fluorescence detection. ${ }^{[1]}$ The assay was performed under the following conditions: (1) chromato bar: Dima C18 (4.6 $\times 150 \mathrm{~mm}, 5 \mu \mathrm{m})$; (2) mobile phase: $0.07 \mathrm{~mol} / \mathrm{L}$, acetic acid buffer solution (containing $2 \%$ methanol); (3) flow rate: $0.8 \mathrm{~mL} / \mathrm{min}$; (4) sample size: $15 \mu \mathrm{L}$; (5) column temperature: $25^{\circ} \mathrm{C}$; (6) analysis time: $15 \mathrm{~min}$; (7) fluorescence detection condition: excitation wavelength $390 \mathrm{~nm}$, emission wavelength $470 \mathrm{~nm}$, and photomultiplier tube (PMT) of 12. All the technological indexes such as precision, recovery rate, stability, sensitivity, and linear range met the criteria for biological sample detection.

\section{Statistical analysis}

The primary endpoint of this report is incident stroke. In addition, stroke death was analyzed similarly. The key predictors of interest are baseline plasma tHcy levels and blood pressure. Both were analyzed as continuous and categorical variables. Because the distribution of plasma tHcy was skewed, it was logarithmically transformed in the analysis. Geometric means are presented in Table 1. On the basis of the exploratory analysis of the relationship between tHcy levels and incident stroke and stroke death, three categories of plasma tHcy levels were used in the analysis: low $(<10 \mu \mathrm{mol})$, moderate $(\geq 10 \mu \mathrm{mol}$ but $<20 \mu \mathrm{mol})$, and high $(\geq 20 \mu \mathrm{mol})$. The cutoff tHcy level of $<10 \mu \mathrm{mol}$ is recommended by the American Heart Association and the American Stroke Association Council on Stroke ${ }^{[25,26]}$.

Hypertension was used as a binary variable (yes/no) and defined as diastolic blood pressure (DBP) $\geq 90 \mathrm{mmHg}$ and/ or systolic blood pressure (SBP) $\geq 140 \mathrm{mmHg}$. Student's $t$ and chi-squared tests were used to assess differences between cases and controls among major baseline characteristics, including baseline plasma tHcy levels and SBP and DBP. Multiple logistic regression was used to 
Table 1: Epidemiological and clinical characteristics of cases and controls at the baseline by stroke endpoints ${ }^{\dagger}$

\begin{tabular}{|c|c|c|c|c|}
\hline \multirow[t]{3}{*}{ Variables } & \multicolumn{2}{|l|}{ Incident stroke } & \multicolumn{2}{|l|}{ Stroke death } \\
\hline & Matched controls & Cases & Matched controls & Cases \\
\hline & Mean $\pm \operatorname{SD}(n)$ & Mean $\pm \operatorname{SD}(n)$ & Mean $\pm \operatorname{SD}(n)$ & Mean $\pm \operatorname{SD}(n)$ \\
\hline$n$ & 179 & 179 & 121 & 121 \\
\hline Age, years & $58.1 \pm 11.1(179)$ & $58.4 \pm 10.8(179)$ & $58.7 \pm 11.9(121)$ & $59.4 \pm 11.3(121)$ \\
\hline BMI, $\mathbf{k g} / \mathrm{m}^{2}$ & $20.8 \pm 2.6(179)$ & $21.6 \pm 3.0(175)$ & $20.6 \pm 2.6(121)$ & $21.3 \pm 3.0(119)^{*}$ \\
\hline tHcy, $\mu \mathrm{mol} / \mathrm{L}$ & $15.5 \pm 7.1(175)$ & $18.2 \pm 13.5(175)^{*}$ & $15.8 \pm 7.9(118)$ & $19.6 \pm 15.0(118)^{*}$ \\
\hline \multirow[t]{2}{*}{ Natural logarithm of tHcy } & $2.7 \pm 0.3(175)$ & $2.8 \pm 0.5(175)^{*}$ & $2.7 \pm 0.4(118)$ & $2.8 \pm 0.5(118)^{*}$ \\
\hline & $n(\%)$ & $n(\%)$ & $n(\%)$ & $n(\%)$ \\
\hline Gender (males) & $127(70.9)$ & $125(69.8)$ & $90(74.4)$ & $90(74.4)$ \\
\hline Non smoking ${ }^{\ddagger}$ & $91(52.6)$ & $93(54.1)$ & $62(51.2)$ & $60(49.6)$ \\
\hline Nonalcohol drinking & $50(28.9)$ & $44(25.6)$ & $33(27.3)$ & $30(24.8)$ \\
\hline \multicolumn{5}{|l|}{ Hypertension } \\
\hline Yes & $90(50.3)$ & $139(77.7)^{* *}$ & $59(48.8)$ & $92(76.0)^{* *}$ \\
\hline No & $89(49.7)$ & $34(19.0)$ & $62(51.2)$ & $26(21.5)$ \\
\hline Missing & $0(0.0)$ & $6(3.4)$ & $0(0.0)$ & $3(2.5)$ \\
\hline \multicolumn{5}{|l|}{ Dyslipidemia } \\
\hline Yes & $23(12.8)$ & $32(17.9)$ & $15(12.4)$ & $19(15.7)$ \\
\hline No & $91(50.8)$ & $80(44.7)$ & $56(46.3)$ & $50(41.3)$ \\
\hline Missing & $65(36.3)$ & $67(37.4)$ & $50(41.3)$ & $52(43.0)$ \\
\hline \multicolumn{5}{|l|}{ Blood glucose abnormal } \\
\hline Yes & $22(12.3)$ & $17(9.5)$ & $12(9.9)$ & $10(8.3)$ \\
\hline No & $88(49.2)$ & $90(50.3)$ & $56(46.3)$ & $56(46.3)$ \\
\hline Missing & $69(38.5)$ & $72(40.2)$ & $53(43.8)$ & $55(45.5)$ \\
\hline
\end{tabular}

†Continuous variables tested using Student's t tests and categorical variables tested using chi-squared tests; $P<0.05,{ }^{* *} P<0.01$; ${ }^{\ddagger}$ Nonsmoking was defined as never smoking. ${ }^{\ddagger}$ Nonalcohol drinking was defined as never alcohol drinking.

Table 2: Relative odds of stroke endpoints by baseline plasma tHcy levels.

\begin{tabular}{|c|c|c|c|c|c|c|c|c|}
\hline \multirow[t]{2}{*}{ tHcy category } & \multirow[t]{2}{*}{$n$} & \multirow{2}{*}{$\begin{array}{l}\text { Cases } \\
n(\%)\end{array}$} & \multicolumn{3}{|c|}{ Unadjusted } & \multicolumn{3}{|c|}{ Adjustedt } \\
\hline & & & $\overline{\text { OR }}$ & $95 \% \mathrm{Cl}$ & $P$ & OR & $95 \% \mathrm{Cl}$ & $P$ \\
\hline \multicolumn{9}{|l|}{ Odds of incident stroke } \\
\hline$\overline{\mathrm{tHcy}}<10 \mu \mathrm{mol} / \mathrm{L}$ & 36 & $13(36.1)$ & 1.0 & --- & --- & 1.0 & --- & --- \\
\hline $10 \leq \mathrm{tHcy}<20 \mu \mathrm{mol} / \mathrm{L}$ & 262 & $128(48.9)$ & 1.7 & $0.8-3.5$ & 0.154 & 1.7 & $0.8-3.7$ & 0.186 \\
\hline $\mathrm{tHcy} \geq 20 \mu \mathrm{mol} / \mathrm{L}$ & 52 & $34(65.4)$ & 3.3 & $1.4-8.1$ & 0.008 & 3.1 & $1.2-8.6$ & 0.022 \\
\hline$P$ for Trend & & & & & 0.006 & & & 0.020 \\
\hline \multicolumn{9}{|l|}{ Odds of stroke death } \\
\hline thcy $<10 \mu \mathrm{mol} / \mathrm{L}$ & 27 & $7(25.9)$ & 1.0 & --- & --- & 1.0 & +-- & --- \\
\hline $10 \leq \mathrm{tHcy}<20 \mu \mathrm{mol} / \mathrm{L}$ & 166 & $83(50.0)$ & 2.9 & $1.1-7.1$ & 0.024 & 2.8 & $1.1-7.4$ & 0.036 \\
\hline $\mathrm{tHcy} \geq 20 \mu \mathrm{mol} / \mathrm{L}$ & 45 & $30(66.7)$ & 5.7 & $2.0-16.5$ & 0.001 & 5.1 & $1.6-16.4$ & 0.006 \\
\hline$P$ for Trend & --- & --- & & & 0.001 & & & 0.007 \\
\hline
\end{tabular}

tMultivariable logistic models adjusted for age, gender, body mass index, smoking, alcohol drinking, fasting blood lipids, fasting blood glucose, and blood pressure. 
examine the associations of blood pressure [reported as hypertension (yes/no)] and plasma tHcy levels (reported as categories: low $(<10 \mu \mathrm{mol} / \mathrm{L})$, moderate $[\geq 10 \mu \mathrm{mol} / \mathrm{L}$ but $<20 \mu \mathrm{mol} / \mathrm{L})$, and high $(\geq 20 \mu \mathrm{mol} / \mathrm{L})]$ with the odds of stroke (incident stroke or stroke death). The dose-response relationship between tHcy levels and incident stroke and stroke death was further examined by analyzing tHcy level as a continuous variable and performing a linear trend test.

Finally, subjects with concomitant hypertension and elevated Hcy ( $\geq 10 \mu \mathrm{mol} / \mathrm{L})$ were defined as having $\mathrm{H}$-type hypertension ${ }^{[27}$. When analyzing tHcy as a binary variable [normal $(\mathrm{tHcy}<10 \mu \mathrm{mol} / \mathrm{L}) /$ high $(\mathrm{tHcy} \geq 10$ $\mu \mathrm{mol} / \mathrm{L})]$, we simultaneously included high plasma tHcy and hypertension as well as an interaction term in the model to examine if they interactively increased the risk of stroke. We also examined the joint effects of H-type hypertension on the risk of stroke by comparing four groups: (1) normal blood pressure/normal tHcy $(\mathrm{tHcy}<10 \mu \mathrm{mol} / \mathrm{L})$ as the reference group, (2) normal blood pressure/high tHcy,
(3) high blood pressure/normal tHcy, and (4) high blood pressure/high tHcy.

We presented the results with and without adjustment for major covariates including age, gender, BMI, smoking, alcohol drinking, fasting blood lipids, fasting blood glucose, and blood pressure levels. Two-sided P values of $<0.05$ were chosen to indicate statistical significance. The analyses were performed with SAS software, version 8.0 (SAS Institute), and SigmaPlot for Windows, version 10.0 (Systat Software, Inc).

\section{RESULTS}

\section{Baseline characteristics of cases and controls}

The baseline characteristics of the study participants by incident stroke and stroke death are presented in Table 1. For both endpoints, mean tHcy levels and prevalence of hypertension were significantly higher among cases than among controls. There were no significant differences

Table 3: Relative odds of stroke endpoints by baseline hypertension status.

\begin{tabular}{|c|c|c|c|c|c|c|c|c|}
\hline \multirow[t]{2}{*}{ Hypertension } & \multirow[t]{2}{*}{$n$} & \multirow{2}{*}{$\begin{array}{l}\text { Cases } \\
n(\%)\end{array}$} & \multicolumn{3}{|c|}{ Unadjusted } & \multicolumn{3}{|c|}{ Adjustedt } \\
\hline & & & OR & $95 \% \mathrm{Cl}$ & $P$ & OR & $95 \% \mathrm{Cl}$ & $P$ \\
\hline \multicolumn{9}{|c|}{ Odds of incident stroke } \\
\hline No & 123 & $34(27.6)$ & 1.0 & --- & +-- & 1.0 & --- & --- \\
\hline Yes & 229 & $139(60.7)$ & 3.5 & $2.2-5.6$ & $<0.001$ & 3.8 & $2.3-6.4$ & $<0.001$ \\
\hline \multicolumn{9}{|c|}{ Odds of stroke death } \\
\hline No & 88 & $26(29.5)$ & 1.0 & --- & --- & 1.0 & --- & --- \\
\hline Yes & 151 & $92(60.9)$ & 3.4 & $1.9-5.8$ & $<0.001$ & 3.2 & $1.8-6.0$ & $<0.001$ \\
\hline
\end{tabular}

†Multivariable logistic models adjusted for age, gender, body mass index, smoking, alcohol drinking, fasting blood lipids, fasting blood glucose, and tHcy levels. $¥$ Normal blood pressure is defined as systolic blood pressure of $<140 \mathrm{mmHg}$ and diastolic blood pressure of $<90$ mmHg; hypertension is defined as systolic blood pressure of $\geq 140 \mathrm{mmHg}$ and/or diastolic blood pressure of $\geq 90 \mathrm{mmHg}$.

Table 4: Joint associations of blood pressure and plasma tHcy levels with odds of stroke endpoints.

\begin{tabular}{|c|c|c|c|c|c|c|c|c|c|}
\hline \multirow[t]{2}{*}{ Hypertension } & \multirow{2}{*}{$\begin{array}{l}\text { tHcy } \\
\text { category }\end{array}$} & \multirow[t]{2}{*}{$n$} & \multirow{2}{*}{$\frac{\text { Cases }}{n(\%)}$} & \multicolumn{3}{|c|}{ Unadjusted } & \multicolumn{3}{|c|}{ Adjusted $^{\dagger}$} \\
\hline & & & & OR & $95 \% \mathrm{Cl}$ & $P$ & OR & $95 \% \mathrm{Cl}$ & $P$ \\
\hline \multicolumn{10}{|c|}{ Odds of incident stroke } \\
\hline No & Normal & 17 & $2(11.8)$ & 1.0 & -- & --- & 1.0 & --- & 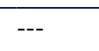 \\
\hline No & High & 104 & $31(29.8)$ & 3.8 & $0.8-17.5$ & 0.087 & 3.5 & $0.7-16.5$ & 0.109 \\
\hline Yes & Normal & 19 & $11(57.9)$ & 10.3 & $1.8-58.4$ & 0.008 & 9.7 & $1.7-56.4$ & 0.011 \\
\hline Yes & High & 204 & $125(61.3)$ & 11.9 & $2.6-53.3$ & 0.001 & 12.7 & $2.8-58.0$ & 0.001 \\
\hline \multicolumn{10}{|c|}{ Odds of stroke death } \\
\hline No & Normal & 15 & $2(13.3)$ & 1.0 & --- & --- & 1.0 & --- & --- \\
\hline No & High & 72 & $24(33.3)$ & 3.7 & $0.8-17.4$ & 0.104 & 3.6 & $0.7-17.3$ & 0.113 \\
\hline Yes & Normal & 12 & $5(41.7)$ & 4.6 & $0.7-30.4$ & 0.109 & 4.3 & $0.6-28.6$ & 0.135 \\
\hline Yes & High & 136 & $86(63.2)$ & 11.2 & $2.4-51.6$ & 0.002 & 11.7 & $2.5-54.7$ & 0.002 \\
\hline
\end{tabular}

†Multivariable logistic models adjusted for age, gender, BMI, smoking, alcohol drinking, fasting blood lipids, and fasting blood glucose levels. ${ }^{\ddagger}$ High blood pressure is defined as systolic blood pressure of $\geq 140 \mathrm{mmHg}$ and/or diastolic blood pressure of $\geq 90 \mathrm{mmHg}$; normal blood pressure is defined as systolic blood pressure of $<140 \mathrm{mmHg}$ and diastolic blood pressure of $<90 \mathrm{mmHg}$; ${ }^{\ddagger}$ Normal thcy level is defined as tHcy of $<10 \mu \mathrm{mol} / \mathrm{L}$, high tHcy level is defined as $\mathrm{tHcy}$ of $\geq 10 \mu \mathrm{mol} / \mathrm{L}$. 
between cases and controls in other known risk factors for vascular disease including age, gender, BMI, smoking, alcohol drinking, fasting blood glucose, and dyslipidemia.

\section{Plasma tHcy and stroke}

As shown in Table 2, the odds of incident stroke and stroke death increased with higher levels of tHcy. After adjustment for the major covariates including blood pressure, and using the low tHcy group as the reference, the odds ratios (ORs) [95\% confidence interval (CI)] for the moderate and high tHcy groups, respectively, were $1.7(0.8-3.7)$ and 3.1 (1.2-8.6) for incident stroke and $2.8(1.1-7.4)$ and 5.1 (1.6-16.4) for stroke death. There was also a significant linear trend for incident stroke $(P<0.005)$ and stroke death $(P<0.001)$, suggesting a dose-response relationship.

\section{Blood pressure and stroke}

Table 3 shows that the odds of incident stroke and stroke death were significantly higher among subjects with hypertension relative to those with normal blood pressure. After adjustment for the major covariates including baseline tHcy level, the ORs (95\% CI) were 3.8 (2.3-6.4) for incident stroke and $3.2(1.8-6.0)$ for stroke death.

\section{Joint associations of plasma tHcy levels and hypertension with stroke}

Subjects with concomitant hypertension and elevated Hcy $(\geq 10 \mu \mathrm{mol} / \mathrm{L})$ were defined as having H-type hypertension. Table 4 shows the joint associations of plasma tHcy levels and hypertension with the odds of incident stroke and stroke death. After adjustment for the major covariates and using normal blood pressure/normal tHcy as the reference group, the odds of incident stroke were elevated in subjects with high tHcy levels but normal blood pressure [3.5 (0.7-16.5)] and in subjects with hypertension but normal tHcy levels [9.7 (1.7-56.4)]. Of note, the highest odds were found among subjects with $\mathrm{H}$-type hypertension, that is, with both high tHcy (tHcy $\geq 10 \mathrm{mmol} / \mathrm{L}$ ) and hypertension [12.7 (2.8-58.0)]. A similar pattern was found for stroke death.

Finally, to test for potential interaction, we simultaneously included plasma tHcy (both as a categorical and continuous variable) and hypertension as well as an interaction term in the model. Our results indicate that there is no significant interaction between plasma tHcy and hypertension on the risk of stroke and stroke death. However, plasma tHcy and hypertension appear to act additively on a multiplicative scale to increase the risk of stroke and stroke death.

\section{DISCUSSION}

Our study addresses two important questions that have not been well examined in previous studies, that is, whether hyperhomocysteinemia and hypertension each independently contribute to incident stroke and stroke death and whether they synergistically increase the risk of incident stroke and stroke death in a Chinese population. Using a nested case-control design from a large prospective cohort, we found that hyperhomocysteinemia and hypertension were independent risk factors of incident stroke and stroke death. Furthermore, subjects with both risk factors had the highest risk of incident stroke and stroke death, while subjects with neither risk factor had the lowest risk. The joint association of hyperhomocysteinemia and hypertension with incident stroke and stroke death appears to be additive on a multiplicative scale.

Our findings are consistent with published literature. For example, Nygard et al. ${ }^{[3]}$ prospectively investigated the relation between plasma tHcy levels and mortality among 587 patients with angiographically confirmed CAD. They found that the 5 -year survival rate for patients with hyperhomocysteinemia was significantly lower than that for patients with normal Hcy levels. Wald et al. ${ }^{[6]}$ conducted a meta-analysis on data from 26 studies, with 176,186 subjects and 3,820 vascular disease events in total. Their findings provided strong support that the association between homocysteine and cardiovascular disease is causal. Furthermore, their findings concluded that by increasing folic acid intake and thereby lowering Hcy concentrations by $3 \mathrm{mmol} / \mathrm{L}$ from current levels, the risk of ischemic heart disease was reduced by $16 \%$ (11-20\%), deep vein thrombosis by $25 \%(8-38 \%)$, and stroke by $24 \%(15-33 \%){ }^{[6]}$ Recently, a meta-analysis of nine prospective observational studies ${ }^{[28]}$ composed of 13,284 participants provided convincing evidence that the pooled risk ratios (RRs) of ischemic stroke, hemorrhagic stroke, and recurrent stroke when comparing the group with the highest Hcy level to the group with the lowest Hcy level were 1.69 (95\% CI: 1.29-2.20) , 1.65 (95\% CI: $0.61-4.45)$, and 1.76 (95\% CI: $1.37-2.24)$, respectively. Their conclusive findings showed that elevated Hcy levels are associated with an increased risk for ischemic stroke and recurrent stroke but had no distinct association with hemorrhagic stroke. Likewise, essential hypertension is a well-recognized and prevalent risk factor for cardiovascular disease including stroke ${ }^{[4,29,30]}$. While previous studies have shown that hyperhomocysteinemia and hypertension often coexist ${ }^{[9,31-33]}$, their joint or interactive association with stroke has not been well examined.

Our findings have important clinical and public health implications. First, our prospective data suggest that elevated tHcy is an independent risk factor for incident stroke and stroke death in the Chinese population. In a similar study with the Japanese population, a prospective, nested, case-control study of 11,846 subjects $40-85$ years 
of age was conducted ${ }^{[12]}$. Consistent with our study, their findings showed that the multivariate ORs $(95 \% \mathrm{CI})$ for highest versus lowest quartiles of Hcy after adjustment for important multiple confounding factors were 2.99 (1.51-5.93) for total stroke, 3.89 (1.60-9.46) for ischemic stroke, $3.36(1.27-8.90)$ for lacunar infarction, and 1.63 (0.44-6.00) for hemorrhagic stroke. However, their data did not exhibit an interactive effect for smoking status, hypertensive status, or hyperhomocysteinemia on ischemic stroke risk. Two similar community-based prospective cohort studies were performed in the Chinese populations ${ }^{[13,19]}$. The first was a 12-year prospective cohort study ${ }^{[13]}$, which found that participants with Hcy of $>9.47 \mu \mathrm{mol} / \mathrm{L}$ had a 2.3 -fold risk for cardiovascular events (1.24-4.18) and participants with Hcy of $>11.84 \mu \mathrm{mol} / \mathrm{L}$ had a 2.4fold risk for all-cause death (1.76-3.32); however, Hcy was not significantly related with risk for stroke. The second study was a large prospective cohort of stroke patients with secondary vascular events and mortality ${ }^{[19]}$, which found that when comparing highest and lowest quartile categories, a high Hcy concentration was associated with a 1.74-fold increased risk for stroke recurrence (1.3-2.3) and a 1.75-fold increased risk for all-cause mortality (1.3-2.4). Most recently, a population-based, prospective cohort study among 5,935 participants from 60 communities in the city of Shenzhen, China ${ }^{[34]}$, showed that the hazard ratios $(95 \% \mathrm{CI})$ of ischemic stroke associated with hyperhomocysteinemia (tHcy $\geq 15 \mu \mathrm{mol} / \mathrm{L}$ ) were 2.18 (1.65-2.89) in the total participants, but no risk was shown for coronary heart disease susceptibility. Worldwide, hyperhomocysteinemia is prevalent across populations. For example, in the original Framingham Heart Study cohort aged 67-96 years, Selhub et al. ${ }^{\left[{ }^{[5]}\right.}$ found that about $19 \%$ of participants' plasma Hcy was higher than 16.4 with levels increasing as subject age increased. A published study of the Chinese population aged 35-64 years showed the prevalence of hyperhomocysteinemia (tHcy $\geq 16 \mathrm{mmol} / \mathrm{L}$ ) in this population to be $17.7 \%$ (25.9\% in males and $9.9 \%$ in females) ${ }^{[36]}$.

Second, our data shows that hypertension is an independent risk factor for stroke and acts additively with hyperhomocysteinemia to increase the risk of stroke. As with hyperhomocysteinemia, hypertension is highly prevalent in Chinese populations. According to a recent national survey, at least $18.8 \%$ of Chinese adults are hypertensive ${ }^{[37]}$.

Third, given our study's findings, for primary stroke prevention in the Chinese population, it would be prudent to reduce the prevalence of hyperhomocysteinemia and hypertension in this population. In a previous meta-analysis conducted by Wang et al. ${ }^{[38]}$, we showed an inverse trend between Hcy reduction and RR of stroke. The observed dose-response relationship provides further evidence that Hcy reduction may be one effective way to reduce stroke risk.

In interpreting our findings, one important limitation needs to be addressed. We were unable to distinguish stroke type because of the lack of imaging data. The proportion of hemorrhagic versus ischemic stroke could influence the stroke death rate. The relatively high rate of mortality because of stroke in this population suggests this cohort may have experienced a high proportion of hemorrhagic stroke ${ }^{[39]}$.

In summary, this large-scale prospective study investigated the independent and joint associations of plasma Hcy levels and hypertension with incident stroke and stroke death in Chinese adults. This study provides strong evidence that H-type hypertension is comprised of two independent, modifiable risk factors, which act additively to increase the risk of incident stroke and stroke death. Our data suggest that those with $\mathrm{H}$-type hypertension in the Chinese population may particularly benefit from Hcy-lowering therapy along with standard antihypertensive therapy.

\section{Acknowledgement}

We gratefully acknowledge the assistance and cooperation of the faculty and staff of the Anhui Medical University and thank all of the participants in our study. This study was conducted in accordance with the current regulations of the People's Republic of China.

\section{Conflicts of Interest}

The authors declare no conflicts of interest.

\section{Source of Support}

This study was supported by the National Science and Technology Major Projects Specialized for "Major New Drugs Innovation and Development" during the 12th Five-Year Plan Period (Grant No. zx09101105), and the National Natural Science Foundation of China (Grant No. 81473052, 81373484, 81141116 and 30700454$)$.

\section{REFERENCES}

1. Anderson JL, Muhlestein JB, Horne BD, Carlquist JF, Bair TL, Madsen TE, et al. Plasma homocysteine predicts mortality independently of traditional risk factors and C-reactive protein in patients with angiographically defined coronary artery disease. Circulation 2000;102:1227-32.

2. Boushey CJ, Beresford SA, Omenn GS, Motulsky AG. A quantitative assessment of plasma homocysteine as a risk factor for vascular disease. Probable benefits of increasing folic acid intakes. JAMA 1995;274:104957. 
3. Nygard O, Nordrehaug JE, Refsum H, Ueland PM, Farstad M, Vollset SE. Plasma homocysteine levels and mortality in patients with coronary artery disease. N Engl J Med 1997;337:230-6.

4. Homocysteine Studies Collaboration. Homocysteine and risk of ischemic heart disease and stroke: a meta-analysis. JAMA 2002;288:2015-22.

5. Davey Smith G, Ebrahim S. 'Mendelian randomization': can genetic epidemiology contribute to understanding environmental determinants of disease? Int J Epidemiol 2003;32:1-22.

6. Wald DS, Law M, Morris JK. Homocysteine and cardiovascular disease: evidence on causality from a meta-analysis. BMJ 2002;325:1202.

7. Casas JP, Bautista LE, Smeeth L, Sharma P, Hingorani AD. Homocysteine and stroke: evidence on a causal link from Mendelian randomization. Lancet 2005;365:224-32.

8. Ueland PM, Refsum H, Brattstrom L. Plasma homocysteine and vascular disease. In: Francis RB Jr, ed. Atherosclerotic Cardiovascular Disease, Hemostasis and Endothelial Function. New York, NY: Marcel Dekker Inc;. 1992:183-236.

9. Graham IM, Daly LE, Refsum HM, Robinson K, Brattström LE, Ueland $\mathrm{PM}$, et al. Plasma homocysteine as a risk factor for vascular disease. The European Concerted Action Project. JAMA 1997;277:1775-81.

10. Brattstrom L, Wilcken DE, Ohrvik J, Brudin L. Common methylenetetrahydrofolate reductase gene mutation leads to hyperhomocysteinemia but not to vascular disease: the result of a meta-analysis. Circulation 1998;98:2520-6.

11. Charpiot P, Bescond A, Augier T, Chareyre C, Fraterno M, Rolland PH, et al. Hyperhomocysteinemia induces elastolysis in minipig arteries: structural consequences, arterial site specificity and effect of captoprilhydrochlorothiazide. Matrix Biol 1998;17:559-74.

12. Iso $\mathrm{H}$, Moriyama $\mathrm{Y}$, Sato $\mathrm{S}$, Kitamura A, Tanigawa T, Yamagishi $\mathrm{K}$, et al. Serum total homocysteine concentrations and risk of stroke and its subtypes in Japanese. Circulation 2004;109:2766-72.

13. Sun Y, Chien KL, Hsu HC, Su TC, Chen MF, Lee YT. Use of serum homocysteine to predict stroke, coronary heart disease and death in ethnic Chinese. 12-year prospective cohort study. Circ J 2009; 73:1423-30.

14. Verhoef P, Hennekens CH, Malinow MR, Kok FJ, Willett WC, Stampfer MJ. A prospective study of plasma homocyst(e)ine and risk of ischemic stroke. Stroke 1994;25:1924-30.

15. Fallon UB, Elwood P, Ben-Shlomo Y, Ubbink JB, Greenwood R, Smith GD. Homocysteine and ischaemic stroke in men: the Caerphilly study. J Epidemiol Community Health 2001;55:91-6.

16. Boushey CJ, Beresford SA, Omenn GS, Motulsky AG. A quantitative assessment of plasma homocysteine as a risk factor for vascular disease. Probable benefits of increasing folic acid intakes. J Am Med Assoc 1995;274:1049-57.

17. Moller J, Nielsen GM, Tvedegaard KC, Andersen NT, Jorgensen PE. A meta-analysis of cerebrovascular disease and hyperhomocysteinaemia. Scand J Clin Lab Invest 2000;60:491-9.

18. Collaboration HS. Homocysteine and risk of ischemic heart disease and stroke: a meta-analysis. J Am Med Assoc 2002;288:2015-22.

19. Zhang W, Sun K, Chen J, Liao Y, Qin Q, Ma A, et al. High plasma homocysteine levels contribute to the risk of stroke recurrence and allcause mortality in a large prospective stroke population. Clin Sci (Lond) 2010;118:187-94.

20. Jones DW, Chambless LE, Folsom AR, Heiss G, Hutchinson RG, Sharrett $\mathrm{AR}$, et al. Risk factors for coronary heart disease in African Americans: the atherosclerosis risk in communities study, 1987-1997. Arch Intern Med 2002;162:2565-71.

21. Kuller LH, Arnold AM, Psaty BM, Robbins JA, O'Leary DH, Tracy RP, et al. 10-year follow-up of subclinical cardiovascular disease and risk of coronary heart disease in the Cardiovascular Health Study. Arch Intern Med 2006;166:71-8.

22. Seshadri S, Beiser A, Kelly-Hayes M, Kase CS, Au R, Kannel WB, et al. The lifetime risk of stroke: estimates from the Framingham Study. Stroke 2006;37:345-50.
23. Stegmayr B, Asplund K, Kuulasmaa K, Rajakangas AM, Thorvaldsen P, Tuomilehto J. Stroke incidence and mortality correlated to stroke risk factors in the WHO MONICA Project. An ecological study of 18 populations. Stroke 1997;28:1367-74.

24. He J, Klag MJ, Wu Z, Whelton PK. Stroke in the People's Republic of China. II. Meta-analysis of hypertension and risk of stroke. Stroke 1995;26:2228-32.

25. Malinow MR, Bostom AG, Krauss RM. Homocyst(e)ine, diet, and cardiovascular diseases: a statement for healthcare professionals from the Nutrition Committee, American Heart Association. Circulation 1999;99:178-82.

26. Sacco RL, Adams R, Albers G, Alberts MJ, Benavente O, Furie K, et al. Guidelines for prevention of stroke in patients with ischemic stroke or transient ischemic attack: a statement for healthcare professionals from the American Heart Association/American Stroke Association Council on Stroke: co-sponsored by the Council on Cardiovascular Radiology and Intervention: the American Academy of Neurology affirms the value of this guideline. Circulation 2006;113:e409-49.

27. Qin X, Huo Y. H-Type hypertension, stroke and diabetes in China: Opportunities for primary prevention. J Diabetes 2015 Sep 1. doi: 10.1111/1753-0407.12333.

28 He Y, Li Y, Chen Y, Feng L, Nie Z. Homocysteine level and risk of different stroke types: a meta-analysis of prospective observational studies. Nutr Metab Cardiovasc Dis. 2014;24:1158-65.

29. Schnyder G, Roffi M, Pin R, Flammer Y, Lange H, Eberli FR, et al. Decreased rate of coronary restenosis after lowering of plasma homocysteine levels. N Engl J Med 2001;345:1593-600.

30. Perry HM Jr, Davis BR, Price TR, Applegate WB, Fields WS, Guralnik $\mathrm{JM}$, et al. Effect of treating isolated systolic hypertension on the risk of developing various types and subtypes of stroke: the Systolic Hypertension in the Elderly Program (SHEP). JAMA 2000;284:465-71.

31. Ye DS, Li Y, Li DY, Qian WH, Yu H, Xia Y, et al. The epidemiologic study of the relationship between plasma homocysteine and hypertension. $J$ Clin Cardiol. 2005;21:536-8.

32. van Guldener C, Nanayakkara PW, Stehouwer CD. Homocysteine and blood pressure. Curr Hypertens Rep 2003;5:26-31.

33. Papandreou D, Rousso I, Makedou A, Arvanitidou M, Mavromichalis I. Association of blood pressure, obesity and serum homocysteine levels in healthy children. Acta Paediatr 2007;96:1819-23.

34. Han L, Wu Q, Wang C, Hao Y, Zhao J, Zhang L, et al. Homocysteine, ischemic stroke, and coronary heart disease in hypertensive patients: a population-based, prospective cohort study. Stroke 2015;46:1777-86.

35. Selhub J, Jacques PF, Wilson PW, Rush D, Rosenberg IH. Vitamin status and intake as primary determinants of homocysteinemia in an elderly population. JAMA 1993;270:2693-8.

36. Hao L, Ma J, Zhu J, Stampfer MJ, Tian Y, Willett WC, et al. High prevalence of hyperhomocysteinemia in Chinese adults is associated with low folate, vitamin B-12, and vitamin B-6 status. J Nutr 2007;137:407-13.

37. Committee of Guidelines for Hypertension Prevention and Control. Guidelines for Hypertension Prevention and Control in China 2005. Beijing: People's Medical Publishing House, 2015: 1-47.

38. Wang X, Qin X, Demirtas H, Li J, Mao G, Huo Y, et al. Efficacy of folic acid supplementation in stroke prevention: a meta-analysis. Lancet 2007;369:1876-82.

39. Rosamond W, Flegal K, Furie K, Go A, Greenlund K, Haase N, et al. Heart disease and stroke statistics--2008 update: a report from the American Heart Association Statistics Committee and Stroke Statistics Subcommittee. Circulation 2008;117:e25-146.

How to cite this article: Li J, Jiang S, Zhang Y, Tang G, Wang Y, Mao G, Li Z, Xu X, Wang B, Huo Y. H-type hypertension and risk of stroke in Chinese adults: A prospective, nested case-control study. J Transl Intern Med 2015; 3: 171-178. 